\title{
BM] Global Heatth What kind of evidence do we need to strengthen primary healthcare in the 21st century?
}

\author{
Lisa R Hirschhorn, ${ }^{\oplus 1,2}$ Etienne V Langlois, ${ }^{\ominus 3}$ Asaf Bitton, ${ }^{\oplus 45}$ Abdul Ghaffar ${ }^{\odot}$
}

To cite: Hirschhorn LR, Langlois EV, Bitton A, et al. What kind of evidence do we need to strengthen primary healthcare in the 21st century?BMJ Global Health 2019;4:e001668. doi:10.1136/ bmjgh-2019-001668

Received 24 April 2019 Accepted 1 May 2019

Check for updates

(C) Author(s) (or their employer(s)) 2019. Re-use permitted under CC BY-NC. No commercial re-use. See rights and permissions. Published by BMJ.

${ }^{1}$ Medical Social Sciences, Northwestern University Feinberg School of Medicine, Chicago, Illinois, USA ${ }^{2}$ Northwestern University Feinberg School of Medicine, Chicago, Illinois, USA

${ }^{3}$ Alliance for Health Policy and Systems Research, World Health Organization, Geneva, Switzerland

${ }^{4}$ Ariadne Labs, Boston, Massachusetts, USA

${ }^{5}$ Division of General Medicine, Brigham and Women's Hospital, Boston, Massachusetts, USA

\section{Correspondence to} Lisa R Hirschhorn; lisa.hirschhorn@northwestern. edu
The increased recognition of the critical importance of quality primary healthcare (PHC) to achieve effective universal health coverage (UHC) and contribute to the sustainable development goals (SDGs) has reignited global interest in strengthening PHC systems and delivery. ${ }^{1}$ However, this focus has also highlighted the implementation gaps between evidence-informed interventions and their delivery to all people everywhere. These gaps include how to better measure PHC to identify and address challenges and how to adapt and scale effective interventions to bridge three main transitions: evidence to policy, policy to implementation and implementation to system quality and its maintenance. Recent global evidence has shown that system quality failures are now responsible for more deaths than lack of access to care, and greater still than the burden of HIV, tuberculosis and malaria combined. ${ }^{2}$

While there is a growing attention to measuring and improving access to quality primary care, the state of $\mathrm{PHC}$ research in low-income and middle-income countries (LMICs) is currently fragmented, uncoordinated and underfinanced. Most PHC research in LMICs comes from a few high-performing middle-income countries; entire regions of the world have little comparable and available PHC systems, quality and outcome data. ${ }^{3}$ Furthermore, a large proportion of PHC research pertaining to LMICs is still conducted and led by high-income country researchers, raising ethical and practical issues of ownership and research relevance. ${ }^{4}$ Too often this work is focused on measuring and improving inputs to care-including supplies, infrastructure and financing-while ignoring the core functions of service delivery as experienced by users of the system, and the upstream challenges of PHC policymaking and PHC system reforms. While a plethora of research exists on different facets of PHC globally, the confluence of a global agenda on PHC revitalisation is generating an urgent need to prioritise disparate research efforts. Further efforts are needed to advance the field of PHC research around prioritised, targeted and policy-relevant questions linked to the organisation and provision of equitable care. We also need to understand better how to ensure the core service delivery functions of PHC are linked to desired outcomes.

In response to this fragmented evidence and surging demand, the Bill and Melinda Gates Foundation through the Primary Healthcare Performance Initiative (PHCPI) funded Ariadne Labs and the Alliance for Health Policy and Systems Research to conduct a scoping review of the state of the knowledge on PHC in LMICs and provide recommendations for a new PHC research consortium (RC). ${ }^{5}$ This global RC would aim to prioritise a research agenda for PHC and then catalyse this research informed and led by LMICs policymakers, researchers and implementers. Recognising the challenges to uptake of research results, the RC would actively foster the uptake of priority PHC research to support policy and practice while strengthening the capacities of LMIC stakeholders to conduct and use PHC research.

The work on this consortium started with an in-depth review of the literature over the last two decades on PHC delivery in LMICs based on the PHCPI framework. This scoping review is published in the supplement and explores the evidence on PHC in LMIC over the last decade in order to identify critical areas where new knowledge is needed on how the goals of strengthening PHC to reach UHC can be achieved and sustained across a wide range of settings. ${ }^{3}$ The review identified topics relevant to PHC that are well studied, geographic regions where research has been done and major holes in the evidence landscape. The work also identified a need for 
an expanded and more coordinated effort for LMIC-led research on PHC system strengthening, including implementation research on PHC policy and PHC interventions to meet identified country and regional knowledge priorities.

The review was used as a foundational document for a 2017 convening of experts in PHC delivery, research and policy who used the review to prioritise key areas of PHC where gaps exist and new research is needed. ${ }^{3}$ This convening identified four priority research areas with key critical gaps in measurement and improvement knowledge: quality, safety and performance management; PHC policies and governance; organisation and models of care and PHC financing. Ariadne Labs issued a competitive request for proposals in each area to build an evidence gap map and prioritise research questions. Ariadne Labs selected six organisations, five based in LMICs, to explore more deeply these existing knowledge gaps and, through a prioritisation process, identify high-impact questions relevant to their topic areas and region. Among the six organisations, this work engaged a wide range of stakeholders from over 60 countries, including many end-users of research. The grantees also developed preliminary research implementation plans to address how some of these questions might be answered.

In order to achieve the aims of this nascent PHC RC, an organisational structure for conducting this type of networked, prioritised research must be built and funded. The supplement includes a novel landscape analysis of different typologies and structures of health-focused research consortia. ${ }^{6}$ The paper identifies three main domains and a number of key decision steps, including the role of a supporting core, leadership and governance, research prioritisation, membership requirements and responsibilities and overall mission. The landscape analysis was an important underpinning of formative work to inform key decisions for the design of this PHC RC.

The results of the work of these organisations are included in this supplement as well as in papers previously published describing different approaches to effectively and rapidly engage a broad range of stakeholders in a prioritisation process to identify areas where research should first be targeted. ${ }^{7-19}$ Across the four areas and the regions studied, authors uniformly found major gaps such as in supervision and managerial capacity, accountability, community-oriented primary care and interventions to limit out-of-pocket costs. These papers point to the need for a comprehensive research agenda designed to produce evidence on the most effective policy, governance, financial, care delivery models and performance improvement interventions, as well as the strategies to implement them to achieve the goals of improving PHC systems performance in LMICs.

The work of the organisations in the prioritised research areas and resulting evidence gap maps offer a wealth of important insights. Munar and colleagues focused on global evidence for improving performance measurement and management interventions in LMIC
PHC systems, highlighting that the current evidence base contained major gaps including in effective accountability approaches implementation strategies such as continuous quality improvement and supervision. ${ }^{18}$ Fadlallah et al focused on quality, safety and performance management in PHC the Eastern Mediterranean region, where there exists a diverse set of PHC needs and challenges. ${ }^{11}$ They were able to collaboratively convene a range of stakeholders to identify gaps and rank a list of 34 policy-relevant research questions in the region and propose a research implementation plan that emphasises collaborative knowledge generation with policymakers supported for increased demand and use of evidence and impact assessment in order to bridge the evidence policy and practice gaps in this critical area. Rahman and coauthors looked at knowledge gaps for governance, identifying prioritised themes including the role of accountability, public-private partnerships and userprovider communication. ${ }^{17}$

Other works addressed the need for better measurement of primary care service delivery capacity and functions,${ }^{16}$ and evidence gaps and needed new knowledge on effective models and design for community-oriented PHC in Africa. ${ }^{15}$ The World Organization of Family Doctors, led by Goodyear-Smith prioritised areas for further exploration and identified global evidence gaps across LMICs in finance and organisation of primary care. ${ }^{910}$ The work reflects a remarkably broad range of stakeholders, leveraging the robust network of family medicine physicians globally. Prioritised areas for further research included optimal ways to pay for an incentivize PHC delivery and equitable workforce distribution. Colleagues from the George Institute also explored these two areas, focusing on the Asia Pacific region; they describe important new insights into research needed to improve organisation and financing of primary care in the region. ${ }^{12}{ }^{14}$ Prioritised areas included the need for a better understanding of contextual factors and their influence on organisation and financing decisions, interventions to reduce out-of-pocket expenses, financing models designed to strengthen primary healthcare system performance, integration of prevention and control of the growing burden of non-communicable diseases into primary care, and strengthening management capacity. ${ }^{12-14}$

The articles in this supplement represent the collaborative and inclusive work of numerous institutions in high and LMICs, capturing the voices of policymakers, implementers and providers and researchers. These stakeholders have come together to inform and build an RC designed to address current and identify emerging priority knowledge needs in countries and regions working to strengthen PHC. Future work will aim to open the aperture even wider to include patients, their communities and civil society perspectives on the highest value PHC research. This work was designed to build on other efforts to strengthen implementation and policy research capacity and embedded research, ${ }^{20}$ the importance of which is discussed by the commentary by Ejemai 
Eboreime, a researcher and policymaker working across the evidence-policy and implementation divide in PHC. ${ }^{21}$ To succeed, this effort must also remain committed to truly inclusive partnerships, including broad multilateral organisations, country leaders, the community served, and technical experts, as discussed in the editorial by Ghaffar $e t a l .^{22}$ The work done in this formative phase of a PHC RC in an important contribution to catalyse the work needed to help inform where and how work is required to achieve the goals of quality UHC and progress towards the SDGs.

Contributors LRH drafted the manuscript. EVL, $A B$ and AG provided critical revisions. All authors contributed intellectual content, edited the manuscript and approved the final version for submission.

Funding Funding for this research was provided by the Bill and Melinda Gates Foundation.

Competing interests None declared.

Patient consent for publication Not required.

Provenance and peer review Not commissioned; internally peer reviewed.

Data availability statement № additional data are available.

Open access This is an open access article distributed in accordance with the Creative Commons Attribution Non Commercial (CC BY-NC 4.0) license, which permits others to distribute, remix, adapt, build upon this work non-commercially, and license their derivative works on different terms, provided the original work is properly cited, appropriate credit is given, any changes made indicated, and the use is non-commercial. See: http://creativecommons.org/licenses/by-nc/4.0/.

\section{REFERENCES}

1. World Health Organization,, UNICEF. Declaration of Astana. World Health Organization and the United Nations Children's Fund, 2018.

2. Kruk ME, Gage AD, Arsenault C, et al. High-quality health systems in the sustainable development goals era: time for a revolution. Lancet Glob Health 2018;6:e1196-252.

3. Bitton A, Fifield J, Ratcliffe HL, et al. Primary health care system performance in low and middle-income countries: a scoping review of the evidence from 2010 to 2017. BMJ Global Health.

4. World Health Organization. World report on health policy and systems research. World Health Organization, 2017.

5. Veillard J, Cowling K, Bitton A, et al. Better measurement for performance improvement in low- and middle-income countries: the primary health care performance Initiative (PHCPI) experience of conceptual framework development and indicator selection. Milbank Q 2017;95:836-83.
6. VanderZanden A, Langlois E, Ghaffar A, et al. It takes a community: A landscape analysis of global health research consortia. BMJ Global Health.

7. Saif-Ur-Rahman KM, Mamun R, Anwar I. Identifying gaps in primary healthcare policy and governance in low-income and middleincome countries: protocol for an evidence gap MAP. BMJ Open 2019:9:e024316.

8. Munar W, Snilstveit B, Stevenson J, et al. Evidence gap map of performance measurement and management in primary care delivery systems in low- and middle-income countries - study protocol. Gates Open Res 2018;2.

9. Goodyear-Smith F, Bazemore A, Coffman M, et al. A systematic assessment for investigation of primary care financing in low- and middle-income countries. BMJ Global Health.

10. Goodyear-Smith F, Bazemore A, Coffman M, et al. Research gaps in the organisation of primary health care in low- and middle-income countries and ways to address them: a mixed methods approach. BMJ Global Health.

11. Fadlallah R, Bou-Karroum L, El-Jardali F, et al. Quality, safety and performance management in primary health care in Eastern Mediterranean region: from scoping review to research priority setting and implementation plan. BMJ Gloal Health.

12. Dodd R, Palagyi A, Jan S, et al. Organisation of primary health care systems in low- and middle-income countries: review of evidence on what works and why in the Asia-Pacific region. BMJ Global Health.

13. Palagyi A, Dodd R, Jan S, et al. Organisation of primary health care in the Asia-Pacific region: developing a prioritised research agenda. BMJ Global Health.

14. Angell B, Dodd R, Palagyi A, et al. Primary health care financing interventions: a systematic review and development of a stakeholder-driven agenda for the Asia-Pacific region. BMJ Global Health.

15. Mash R, Ray S, Essuman A, et al. Community oriented primary care in the sub-Saharan Africa context: a scoping review of different models, their effectiveness and feasibility. BMJ Global Health.

16. Bresick G, Christians F, Makwero M, et al. Primary health care performance: a scoping review of the current state of primary care performance measurement in Africa. BMJ Global Health.

17. Saif-Ur-Rahman KM, Mamun R, Nowrin I, et al. Primary health care policy and governance in low and middle-income countries: an evidence gap MAP. BMJ Global Health.

18. Munar W, Snilstveit B, Aranda LE, et al. Performance measurement and management in primary healthcare systems in low- and middleincome countries - evidence gap map and implications for the design of a research agenda. BMJ Global Health.

19. Goodyear-Smith F, Bazemore A, Coffman M, et al. Primary care research priorities in Low-and middle-income countries. Ann Fam Med 2019;17:31-5.

20. Ghaffar A, Langlois EV, Rasanathan K, et al. Strengthening health systems through embedded research. Bull World Health Organ 2017; 95 .

21. Eboreime EA. Bridging the "two communities": how an emerging primary health care global research consortium can help achieve universal health coverage in low and middle-income countries. BMJ Global Health.

22. Ghaffar A, Swaminathan S, Gupta A, et al. Three way partnerships fuel primary health care success. BMJ Global Health. 